\title{
Wireless Terahertz Communications
}

\author{
W. Freude ${ }^{\text {1a }}$, T. Harter ${ }^{\text {1ab }}$, S. Ummethala ${ }^{1 a b}$, S. Muehlbrandt ${ }^{1 \text { ab }}$, M. Blaicher ${ }^{1 \text { ab }}$, S. Wolf ${ }^{1 \mathrm{a}}$, M. Weber ${ }^{1 \mathrm{a}}$, \\ F. Boes ${ }^{1 c}$, H. Massler ${ }^{2}$, A. Tessmann ${ }^{2}$, Y. Kutuvantavida ${ }^{1 \mathrm{ab}}$, J. N. Kemal ${ }^{1 \mathrm{a}}$, S. Nellen ${ }^{3}$, \\ L. Hahn $^{1 \mathrm{~b}}$, B. Globisch ${ }^{3}$, M. Walther ${ }^{2}$, T. Zwick ${ }^{1 \mathrm{c}}$, S. Randel ${ }^{1 \mathrm{a}}$, C. Koos $^{1 \mathrm{ab}}$ \\ ${ }^{1}$ Karlsruhe Institute of Technology (KIT IPQ $\left.{ }^{a}, I M T^{b}, I H E^{c}\right)$, Karlsruhe, Germany \\ ${ }^{2}$ Fraunhofer Institute for Applied Solid State Physics (IAF), Freiburg, Germany \\ ${ }^{3}$ Fraunhofer Institute for Telecommunications, Heinrich Hertz Institute (HHI), Berlin, Germany \\ w.freude@kit.edu
}

Over the past years, interest in wireless $\mathrm{THz}$ communications with so-called $\mathrm{T}$-waves has tremendously increased [1]-[4], because the large carrier frequencies in the range $0.2 \mathrm{THz}$ to $0.9 \mathrm{THz}$ support wide signal bandwidths and consequently large data rates. Transmission over hundreds of meters and line rates exceeding $100 \mathrm{Gbit} / \mathrm{s}$ were demonstrated [5]-[10]. Typical atmospheric losses are $0.2 \mathrm{~dB} / 100 \mathrm{~m}$ at $0.2 \mathrm{THz}, 0.5 \mathrm{~dB} / 100 \mathrm{~m}$ at $0.3 \mathrm{THz}, 1.5 \mathrm{~dB} / 100 \mathrm{~m}$ at $0.4 \mathrm{THz}$, and $5 \mathrm{~dB} / 100 \mathrm{~m}$ at $0.9 \mathrm{THz}$. For transmission over a $100 \mathrm{~m}$-distance, however, the unity-gain free-space propagation $\operatorname{loss} a_{\mathrm{dB}}^{(L=100 \mathrm{~m})}=10 \lg (4 \pi L / \lambda)^{2}=120 \mathrm{~dB}(\lambda=1 \mathrm{~mm}, f=0.3 \mathrm{THz})$ dominates. To combat this propagation loss, multiple directional antennas with a high gain per sector can be employed at the base station to boost the reach and the data throughput on transmission and reception.

For generating these large carrier frequencies and for coherently receiving high-speed data, optoelectronic signal processing is the preferred technology [5]. Data provided by a fibre-optical network can be optical-toterahertz converted by photomixing with a III-V uni-travelling-carrier photodiode in a T-wave transmitter (Tx). Coherent wireless multi-carrier $\mathrm{THz}$ transmission over distances of up to $40 \mathrm{~m}$ with a line rate of up to $100 \mathrm{Gbit} / \mathrm{s}$ are demonstrated [6]. An (electronic) in-phase/quadrature (IQ) subharmonic mixer realized as in [7] with a millimetre-wave integrated circuit (MMIC) served as receiver (Rx). An alternative Rx employs a III-V photoconductor which acts as a photomixer for generating the $\mathrm{THz}$ local oscillator frequency electro-optically, and which simultaneously down-converts the received signal to the baseband [11]. As a third option, a novel silicon-plasmonic internal photoemission detector (PIPED) [12] can be used for terahertz-to-electrical baseband conversion and for coherent reception. The PIPED functions also as a photomixer and can be monolithically integrated for realizing a PIPED Tx and a coherent PIPED Rx on a common silicon chip [13]. If both, Tx and $\mathrm{Rx}$, are connected with a co-integrated $\mathrm{THz}$ transmission line, the arrangement can be used as a lab-on-chip for investigating an analyte applied to the transmission line. A fourth Rx type relies on a Schottky barrier diode and digital signal post-processing. Finally, an electro-optical modulator may be driven by a received wireless T-wave signal to transfer the T-wave data transparently to an optical carrier for seamless photonic transport [14]-[17].

The talk will present various $\mathrm{THz}$ transmitter and receiver concepts as well as results from data transmission experiments.

\section{References}

[1] J. Capmany, D. Novak, "Microwave photonics combines two worlds. Nature Photon. 1, 319-330 (2007). (doi:10.1038/nphoton.2007.89)

[2] H.-J. Song, T. Nagatsuma (Eds.), Terahertz technologies. Devices and applications (Pan Stanford Publishing, 2015).

[3] G. Carpintero, L. E. García Muñoz, H. L. Hartnagel, S. Preu, A. V. Räisänen (Eds.), Semiconductor terahertz technology. Devices and systems at room temperature operation (John Wiley \& Sons, 2015).

[4] H. Shams, A. Seeds, "Photonics, fiber and THz wireless communication," Optics \& Photonics News 28, 26-31 (March 2017)

[5] T. Nagatsuma, G. Ducournau, C. C. Renaud, "Advances in terahertz communications accelerated by photonics," Nature Photon. 10, 371-379 (2016). (doi:10.1038/nphoton.2016.65)

[6] S. Koenig et al., "Wireless sub-THz communication system with high data rate," Nature Photon. 7, 977-981 (2013). (doi:10.1038/NPHOTON.2013.275)

[7] I. Kallfass et al., "64 Gbit/s transmission over $850 \mathrm{~m}$ fixed wireless link at $240 \mathrm{GHz}$ carrier frequency. J. Infrared Milli. Terahz. Waves 36, 221-233 (2015). (doi:10.1007/s10762-014-0140-6)

[8] H. Shams et al., "100 Gb/s multicarrier THz wireless transmission system with high frequency stability based on a gain-switched laser comb source,” IEEE Photon. J. 7 (2015) 7902011 (doi:10.1109/JPHOT.2015.2438437)

[9] X. Yu et al., "160 Gbit/s photonics wireless transmission in the $300-500 \mathrm{GHz}$ band," APL Photonics 1 (2016) 081301 (doi:10.1063/1.4960136)

[10] S. Jia et al., "0.4 THz photonic-wireless link with $106 \mathrm{~Gb} / \mathrm{s}$ single channel bitrate,” J. Lightwave Technol. 36, 610-616 (2018). (doi:10.1109/JLT.2017.2776320)

[11] T. Harter et al., "Wireless THz link with optoelectronic transmitter and receiver," arXiv:1901.03140 [physics.app-ph] (2019) (https://arxiv.org/abs/1901.03140)

[12] S. Muehlbrandt et al., "Silicon-plasmonic internal-photoemission detector for 40 Gbit/s data reception," Optica 3, 741-747 (2016). (doi:10.1364/OPTICA.3.000741)

[13] T. Harter et al., "Silicon-plasmonic integrated circuits for terahertz signal generation and coherent detection," Nature Photon. 12, 625633 (2018). (doi:10.1038/s41566-018-0237-x)

[14] S. Ummethala et al., "Terahertz-to-optical conversion using a plasmonic modulator," in Conference on Lasers and Electro-Optics (CLEO 2018), Paper STu3D.4. (doi:10.1364/CLEO_SI.2018.STu3D.4)

[15] S. Ummethala et al., "Wireless transmission at $0 . \overline{3} \mathrm{THz}$ using direct THz-to-optical conversion at the receiver", in 44 th European Conference on Optical Communication (ECOC 2018), Paper We4H.3. (invited)

[16] S. Ummethala et al., "THz-to-Optical conversion in wireless communications using an ultra-broadband plasmonic modulator," arXiv:1812.04121 [physics.app-ph] (2018) (https://arxiv.org/abs/1812.04121)

[17] Y. Salamin, B. Baeuerle et al., "Microwave plasmonic mixer in a transparent fibre-wireless link," Nature Photon. 12, 749-753 (2018). (doi:10.1038/s41566-018-0281-6) 


\section{Repository KITopen}

Dies ist ein Postprint/begutachtetes Manuskript.

\section{Empfohlene Zitierung:}

Freude, W.; Harter, T.; Ummethala, S.; Muehlbrandt, S.; Blaicher, M.; Wolf, S.; Weber, M.; Boes, F.; Massler, H.; Tessmann, A.; Kutuvantavida, Y.; Kemal, J. N.; Nellen, S.; Hahn, L.; Globisch, B.; Walther, M.; Zwick, T.; Randel, S.; Koos, C.

Wireless terahertz communications.

2019. Conference on Lasers and Electro-Optics Europe \& European Quantum Electronics Conference (CLEO/Europe-EQEC): Munich, International Congress Centre (ICM), Germany, 23-27 June 2019.

doi: $\underline{10.5445 / I R / 1000100785}$

Zitierung der Originalveröffentlichung:

Freude, W.; Harter, T.; Ummethala, S.; Muehlbrandt, S.; Blaicher, M.; Wolf, S.; Weber, M.; Boes, F.; Massler, H.; Tessmann, A.; Kutuvantavida, Y.; Kemal, J. N.; Nellen, S.; Hahn, L.; Globisch, B.; Walther, M.; Zwick, T.; Randel, S.; Koos, C. Wireless terahertz communications.

2019. Conference on Lasers and Electro-Optics Europe \& European Quantum Electronics Conference (CLEO/Europe-EQEC): Munich, International Congress Centre (ICM), Germany, 23-27 June 2019, Institute of Electrical and Electronics Engineers (IEEE). doi:10.1109/CLEOE-EQEC.2019.8873098 